\title{
Synthetisch hergestellte Nanomaterialien in Konsum- produkten und deren Verbleib am Ende ihrer Nutzungsphase
}

\author{
F. Part · I. Gruber · K. Hänel · M. Huber-Humer
}

Online publiziert: 14. Dezember 2016

(c) Der/die Autor(en) 2016. Dieser Artikel ist eine Open-Access-Publikation.

\begin{abstract}
Zusammenfassung Seit geraumer Zeit finden künstlich hergestellte und oberflächenmodifizierte Nanomaterialien in diversen Alltagsprodukten Anwendung. Für die gegenständliche Materialflussanalyse wurden 6 Nanokonsumprodukte ausgewählt, die am österreichischen Markt erhältlich sind und vorwiegend metallische Nanomaterialien enthalten (d. h.: Nano-SiO,$-\mathrm{TiO}_{2}$, -Ag oder CdSeQuantenpunkte). Ein Produktbeispiel enthielt Kohlenstoffnanoröhrchen (kurz CNTs). Für die Materialflussanalysen mussten die Nanomaterial-Inputmengen auf Produktebene abgeschätzt werden. Anhand von Produktinformationen sowie wissenschaftlichen Publikationen wurden erste Hochrechnungen des österreichweiten Verbrauchs durchgeführt. Beim Produktbeispiel „TV-Geräte mit Quantenpunkt-Displays“ war die Datengrundlage so dürftig, dass keine Mengenabschätzung möglich war. Dieses Szenario ist aber Beispiel dafür, dass bei vielen Produktkategorien mangelnde bis keine Produktinformationen vorliegen und für diese daher derzeit keine quantitative Abschätzung des Verbleibs von Nanomaterialien in Abfallströmen möglich ist. Die ersten

wie CNTs in Tennisschlägern werden hingegen eher im unteren einstelligen Kilogrammmaßstab eingesetzt. Anhand der Materialflussanalysen lässt sich abschätzen, dass sich metallische/ metalloxidische Nanomaterialien mit hoher Wahrscheinlichkeit im Zuge der Abfallbehandlung in Sekundärabfällen, wie Verbrennungsrückständen, anreichern, welche wiederum größtenteils deponiert werden. Kohlenstoffbasierte Nanomaterialien wie CNTs werden mit hoher Wahrscheinlichkeit während der Abfallverbrennung vollständig oxidiert. Ihre Elimination kann aber nur dann gewährleistet werden, wenn während der Verbrennung eine entsprechend lange Verweildauer bei hohen Temperaturen sichergestellt ist. Die Unsicherheiten bzw. großen Schwankungsbreiten der hochgerechneten Nanomaterialflüsse verdeutlichen, dass es noch große Wissens- und Informationslücken gibt und vor allem entsprechender Forschungsbedarf hinsichtlich des genauen Umweltverhaltens von Nanoprodukten - insbesondere über das Verhalten und potenzielle Freisetzungspfade in der Entsorgungsphase besteht.
\end{abstract} Mengenabschätzungen zeigen, dass Nano- $\mathrm{TiO}_{2}$ bereits im Tonnenmaßstab in Sonnencremen eingesetzt wird (in Österreich ca. 5,4 bis 40,8 t/a). Nano$\mathrm{SiO}_{2}$ in Autoversiegelungen, Nano-Ag in Wandfarben oder Putzlappen so-

DI F. Part $(\bowtie)$

Department für Nanobiotechnologie Institut für Synthetische Bioarchitekturen, Universität für Bodenkultur Wien, Muthgasse 11/II, 1190 Wien, Österreich florian.part@boku.ac.at

DI F. Part · DI I. Gruber · K. Hänel · Univ.-Prof. DI Dr. M. Huber-Humer Department für Wasser,

Atmosphäre und Umwelt, Institut für Abfallwirtschaft, Universität für Bodenkultur Wien, Muthgasse 107, 1190 Wien, Österreich

\section{Synthetically manufactured nanomaterials in consumer products and their fate at the end of service life}

Abstract For some time now, engineered and surface-modified nanomaterials have been used in various everyday products. For the material flow analyses presented in the following paper, six nano-based consumer products were selected that are available on the Austrian market and primarily contain metallic nanomaterials (nano$\mathrm{SiO}_{2}$, $-\mathrm{TiO}_{2}$, - Ag or CdSe quantum dots); one sample product contained carbon nanotubes (CNTs).

For the purpose of the material flow analyses, it was necessary to estimate the amounts of nanomaterial input at the product level. On the basis of avail- able product information and academic publications, initial estimates of consumption in Austria were prepared. In the case of the sample product "televisions with quantum dot displays," there was too little data available to do so. However, this scenario offers valuable proof of the fact that little to no product information is available for many product categories, making it impossible to quantitatively estimate the amount of nanomaterials in waste streams.

The initial estimates show that tons of nano- $\mathrm{TiO}_{2}$ are already used in sun creams (ca. 5.4 to 40.8 t/year in Austria). In contrast, only several kilograms each of nano- $\mathrm{SiO}_{2}$ (in automotive paint sealants), nano-Ag (in indoor paints and washcloths), and CNTs (in tennis rackets) are used each year.

Based on the material flow analyses it can be assumed that, in the course of waste treatment, metallic/metal oxide nanomaterials very likely enrich in secondary waste like combustion residues, which in turn predominantly end up in landfills. Carbon-based nanomaterials like CNTs are most likely completely oxidized in the course of incineration. However, their elimination can only be ensured if they are subjected to high temperatures for a sufficiently long time. The uncertainties/substantial variances in the projected nanomaterial flows clearly show that there are still considerable gaps in our knowledge and available information, and that research regarding the exact environmental fate of nano-products - especially into their behavior and potential emission pathways in the disposal phase is urgently needed.

\section{Einleitung - Nanomaterialien in Konsumprodukten}

Synthetisch hergestellte Nanomaterialien sind Materialien im Ausmaß von 1 bis $100 \mathrm{~nm}$, die sich durch ihre große spezifische Oberfläche sowie ihr weites Oberflächen-zu-Volumen-Verhältnis auszeichnen. Nach internationalen 
Standards (ISO/TS 80004-1:2015) werden Nanomaterialien in Nanoobjekte (i. e. Nanopartikeln, -fasern und -plättchen) sowie in nanostrukturierte Materialien (z. B. nanoskalige Beschichtungen) unterteilt. Aufgrund ihrer Größe sind Nanomaterialien für das menschliche Auge nicht sichtbar und können im Vergleich $\mathrm{zu}$ ihren mikroskaligen Pendants besondere nanospezifische Eigenschaften aufweisen. So ist z. B. nanoskaliges Titandioxid $\left(\mathrm{Nano}-\mathrm{TiO}_{2}\right)$ transparent und wird daher häufig als Additiv in Sonnenschutzcremen eingesetzt. Mikroskaliges $\mathrm{TiO}_{2}$ hingegen ist weiß und wird z. B. als Weißpigment in Papier oder Wandfarben eingesetzt. Cadmiumtellurid (CdTe) ist in (mikroskaliger) Bulkform ein schwarzer Feststoff und wird als Absorbermaterial in Dünnschichtsolarzellen eingesetzt. In nanokristalliner Form kann CdTe als Halbleitermaterial verwendet werden, welches nach elektromagnetischer Anregung sichtbares Licht in unterschiedlichen Farbspektren emittiert. Diese sogenannte Photolumineszenz hängt im Wesentlichen von der Größe dieser Nanokristalle ab: Kleinere Kristalle $(\sim 2 \mathrm{~nm})$ emittieren sichtbares Licht im blauen Spektrum, während größere $(\sim 4 \mathrm{~nm})$ im roten Lichtspektrum emittieren. Auf diesen nanospezifischen Effekt wird bereits in Fernsehgeräten mit Quantenpunkt-basierter Hintergrundbeleuchtung zurückgegriffen (engl. Quantum Dot Displays oder QLED). Im Vergleich zur OLEDTechnologie, welche auf organischen, halbleitenden Materialien basiert, sind Quantenpunkte kostengünstiger herzustellen und weisen zudem eine längere Nutzungsdauer auf (Anikeeva et al. 2009). Um solche Hightech-Produkte in Zukunft umweltfreundlicher zu gestalten, wird seit einigen Jahren an der Herstellung von cadmiumfreien Quantenpunkten gearbeitet (Aboulaich et al. 2011). Nanoskaliges Silber (Nano-Ag) oder Siliziumdioxid (Nano-SiO $\mathrm{N}_{2}$ ) sind weitere Nanomaterialbeispiele, bei welchen die potenziellen Auswirkungen sowie deren Verbleib in der Umwelt noch nicht zur Gänze geklärt sind. Nano-Ag wird aufgrund seiner antibakteriellen Wirkung in diversen Haushaltsprodukten, wie Textilien und Kosmetika, sowie Oberflächenbeschichtungen eingesetzt. Nano-SiO ${ }_{2}$ wird häufig in Wandfarben eingearbeitet, um den sogenannten Lotus-Effekt erzielen zu können.

Im Allgemeinen ist derzeit noch wenig über die möglichen (negativen)
Umweltauswirkungen von Nanomaterialien sowie über die potenziellen Expositionspfade entlang des gesamten Produktlebenszyklus bekannt. Nanomaterialien können aufgrund ihrer Größe leichter als ihre größeren, mikroskaligen Pendants natürliche Barrieren, wie Blut-Hirn-Schranke, Haut- oder Lungengewebe, überschreiten (Krug 2014). Nach derzeitigem Stand des Wissens lassen sich dennoch kaum allgemeine Schlüsse über die toxikologische Wirkung von Nanomaterialien ziehen. So lag z. B. bei zahlreichen toxikologischen Untersuchungen und bisher durchgeführten Studien eine Überdosierung vor oder es wurden toxikologisch-relevante Umwandlungsprozesse, wie die Auflösung von Nanomaterialien in ihre ionischen Bestandteile $(<1 \mathrm{~nm})$, nicht mitberücksichtigt. Derzeit mangelt es vor allem an standardisierten Partikelmessmethoden und Toxizitätstests, die das Fundament hinsichtlich Risikobewertung und Chemikaliensicherheit darstellen (Krug 2014). Über den Umweltverbleib von Nanomaterialien, welche in Konsumprodukten enthalten sind, sowie über deren Verhalten während Abfallbehandlungs- und Entsorgungsprozessen ist noch weniger bekannt (Gazsó et al. 2015). Aus diesem Grund hatte die gegenständliche Studie zum Ziel, den Umweltverbleib von ausgewählten Nanoproduktbeispielen mittels Materialflussanalysen abzuschätzen. Mit solchen quantitativen Analysen können potenzielle Freisetzungspfade bzw. „Hotspots“ entlang des gesamten Produktlebenszyklus (z. B. unbeabsichtigte Freisetzung von Nanomaterialien während der Nutzung oder dem Recycling) sowie abfallwirtschaftliche Senken identifiziert werden.

\section{Vorgehensweise und Methodik}

\subsection{Auswahl der Nanoproduktbeispiele}

Als Nanoprodukte werden jene Produkte bezeichnet, die ein Nanomaterial beinhalten oder z. B. eine Beschichtung in der Dimension von 1 bis $100 \mathrm{~nm}$ haben (AGES 2016; ISO/TS 80004-1:2015). Im Allgemeinen können Nanomaterialien in unterschiedlichen Formen in Konsumprodukten eingesetzt werden. So können Nanomaterialien beispielsweise in Kunststoffe fest eingearbeitet werden oder in Sonnenschutzcremen in dispergierter Form vorkommen. Um die Entwicklung der Nanotechnologie dokumentieren zu können, wurde im
Jahre 2014 die NanoTrust-Datenbank, die von der Österreichischen Akademie der Wissenschaften geführt wird, aktualisiert. Nach dem letzten Update im Juli 2014 wurden in dieser Datenbank mehr als 490 Einträge von Nanoprodukten registriert, die am österreichischen Markt erhältlich sind (Greßler and Gazsó 2014). Die aktualisierte Datenbank diente wiederum als Grundlage, um für die gegenständlichen Materialflussanalysen sechs repräsentative Produktbeispiele auszuwählen. Anhand von durchgeführten ExpertenInneninterviews wurden Auswahlkriterien festgelegt, mithilfe derer die Produktbeispiele gewählt wurden. In Abb. 1 wird der „Kriterien-Entscheidungsbaum“ näher dargestellt. Nach „Priorität 1“ wurden zwei Produktbeispiele ausgewählt, die gemäß gesetzlichen Grundlagen eine Kennzeichnung von nanoskaligen Inhaltsstoffen aufweisen müssen (z. B. gemäß EU-Kosmetikverordnung mit „Nano" gekennzeichnete Sonnencremen). Des Weiteren wurden zwei Produkte aufgrund der vorliegenden hohen Informationsdichte bzw. anhand der Anzahl an wissenschaftlich publizierten Studien ausgewählt („Priorität 2“). Eine weitere Auswahl wurde so getroffen, dass auch Nanomaterialien berücksichtigt werden, welche im Verdacht stehen, eine negative Auswirkung auf die Umwelt und/oder auf abfallwirtschaftliche Prozesse zu haben („Priorität 3“).

Auf Basis der festgelegten Kriterien bzw. Priorität wurden folgende sechs repräsentative Nanoproduktbeispiele ausgewählt, die als Ausgangsszenarien für die gegenständlichen Materialflussanalysen dienten:

- Szenario S1: Nano-TiO ${ }_{2}$ in Sonnencremen,

- Szenario S2A: Nano-Ag in Außenwandfarben,

- Szenario S2B: Nano-Ag in Innenwandfarben,

- Szenario S3: Kohlenstoffnanoröhrchen (kurz CNTs) in Tennisschlägern,

- Szenario S4: Nano-SiO ${ }_{2}$ in Autoversiegelungen,

- Szenario S5A: faserintegriertes NanoAg in Putztüchern,

- Szenario S5B: oberflächenbeschichtetes Nano-Ag in Putztüchern und

- Szenario S6: TV-Geräte mit Quantenpunkt-Displays (z. B. aus CdTe-Halbleiterverbindungen). 


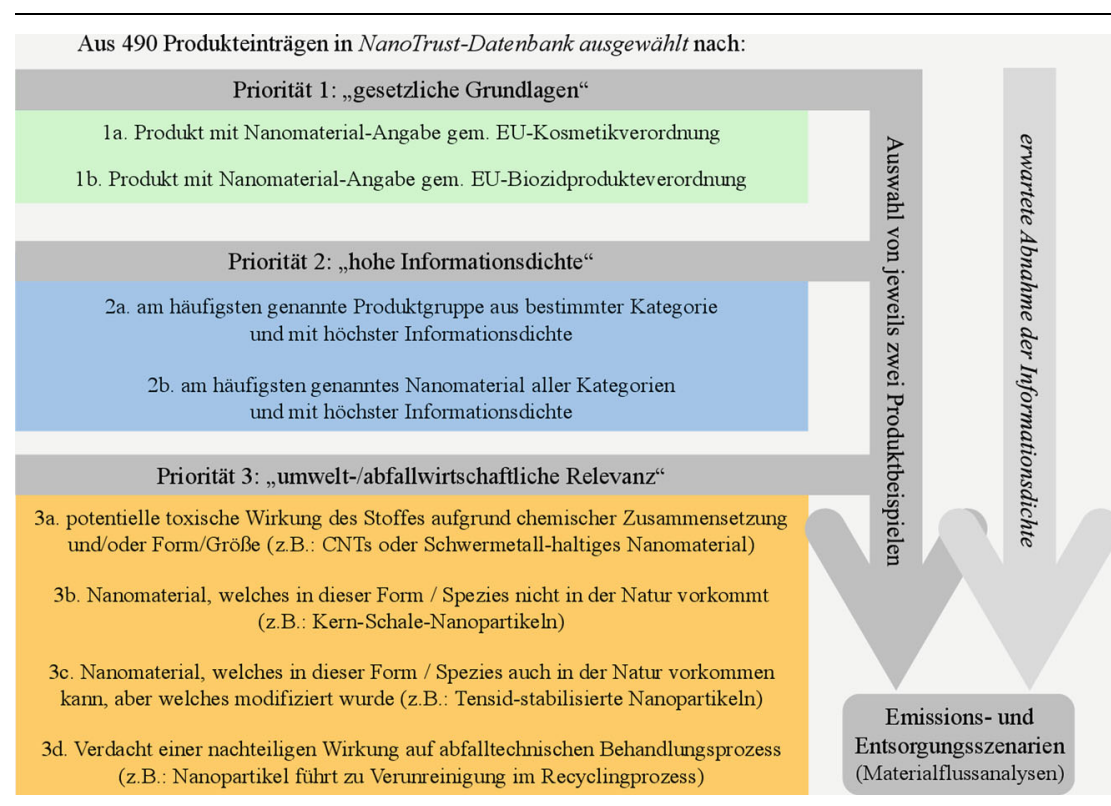

Abb. 1 Kriterien bzw. Prioritäten für die Auswahl von sechs repräsentativen Konsumprodukten für die Materialflussanalysen

\subsection{Inputdaten für Materialflussanalysen}

Um quantitative Aussagen über die potenzielle Verteilung von Nanomaterialien in der Umwelt tätigen zu können, müssen zuerst deren Einsatz- bzw. Produktinhaltsmengen abgeschätzt werden. Hierbei ist anzumerken, dass für die ausgewählten Szenarien keine österreichspezifischen Marktanalysen hinsichtlich Produktionsmengen sowie Marktanteilen vorlagen. Aus diesem Grund wurden für die Hochrechnung der eingesetzten Mengen vorwiegend Daten (über chemische Zusammensetzung, Inhaltsmenge etc.) aus wissenschaftlichen Publikationen und so vorhanden - teilweise aus Produktbroschüren herangezogen. Hinsichtlich „Szenario 1“ wird geschätzt, dass der Marktanteil für Sonnencremen mit anorganischen Inhaltsstoffen (z. B. Nano$\mathrm{TiO}_{2}$ ) ca. $20 \%$ am Gesamtmarkt beträgt (Davis et al. 2010). In einer Studie von Lorenz et al. (2011) wird davon ausgegangen, dass pro Anwendung ca. $8 \mathrm{~g}$ Sonnencreme aufgetragen werden. Mit den weiteren Annahmen, dass $40 \%$ der mitteleuropäischen bzw. der österreichischen Bevölkerung für 20 Tage im Jahr Sonnencreme auftragen (Lorenz et al. 2011) und der Stoffgehalt an $\mathrm{Nano}-\mathrm{TiO}_{2}$ in Sonnencremen ca. 2 bis $15 \%$ beträgt (Davis et al. 2010), wurde ein jährlicher Verbrauch von ca. 5.450 bis $40.840 \mathrm{~kg}$ Nano- $\mathrm{TiO}_{2}$ für Ös- terreich berechnet. Somit konnte für die Materialflussanalyse der niedrigere Verbrauchswert als Inputmenge für das "Minimum-“ und der höhere Berechnungswert für das „Maximum-Szenario S1“ herangezogen werden. Hierbei ist anzumerken, dass diese hohe Schwankungsbreite die Unsicherheiten einer solchen Materialflussanalyse widerspiegelt. Im Rahmen des "Szenarios S2“ wurde angenommen, dass $1 \mathrm{~kg}$ Wandfarbe ca. 6 mg Nano-Ag enthält (Kaegi et al. 2010). ExpertInnen schätzen, dass in Österreich jährlich ca. $45.000 \mathrm{t}$ Wandfarben vertrieben werden, wobei der Marktanteil an Nanomaterial-haltigen Wandfarben derzeit bei geschätzt $0,2 \%$ liegt. Unter Berücksichtigung der Anzahl an österreichischen Herstellern solcher Wandfarben (mindestens 1 bis maximal 5 Hersteller) konnte für Österreich eine Inputmenge von ca. 0,6 bis $3,1 \mathrm{~kg}$ Nano-Ag pro Jahr in Wandfarben berechnet werden. Auffallend ist, dass im Vergleich zu Nano- $\mathrm{TiO}_{2}$ in Sonnenschutzcremen die Einsatzmenge von Nano-Ag in Wandfarben um ein Vielfaches niedriger ist, vermutlich auch deshalb, da im Gegensatz zu Kosmetika für die Produktkategorie Baustoffe keine Produktkennzeichnungspflicht für inkludierte Nanomaterialien besteht. Basierend auf einer Studie von Boldrin et al. (2014) zum weltweiten Aufkommen von CNT-haltigen Tennisschlägern konnte für das „Szenario S3" eine Inputmenge von ca. 0,5 bis
1,4 kg CNTs pro Jahr für Österreich abgeschätzt werden. Für „Szenario 4“ sowie „Szenario 5“ konnten keine relevanten wissenschaftlichen Studien gefunden werden, sondern es musste, sofern vorhanden, auf Herstellerangaben (Befragungen) sowie im Internet veröffentlichte Marktumfragen $\mathrm{zu} \mathrm{Na-}$ noprodukten und Produktbroschüren zurückgegriffen werden. Des Weiteren wurden die getroffenen Annahmen durch eingeholte ExpertInnenmeinungen gestützt (NanoMia 2015). Unter zusätzlicher Verwendung von Daten der STATISTIK AUSTRIA für das Bezugsjahr 2014 wurden so erste, vereinfachte Mengenabschätzungen von Nanomaterialien auf Produktebene dargestellt. So wurde z. B. der österreichweite Jahresverbrauch an Nano- $\mathrm{SiO}_{2}$ in Autoversiegelungen anhand der Anzahl zugelassener Autos - wobei geschätzte $10 \%$ der FahrzeugbesitzerInnen solche Lackschutze verwenden - und anhand eines Marktanteils für selbstreinigende, Nanomaterial-basierte Oberflächen von ca. $3 \%$ abgeschätzt („Szenario 4“). Auf ähnliche Weise wurde der Jahresverbrauch an Nano-Ag in Putzlappen („Szenario 5“) abgeschätzt, wobei die Anzahl an österreichischen Haushalten zur Hochrechnung herangezogen wurde. Mittels solcher Vereinfachungen war es letztendlich möglich, für „Szenario 4" eine Inputmenge von ca. 120 bis $1.200 \mathrm{~kg} \mathrm{Nano-SiO}$ für Autoversiegelungen sowie für „Szenario 5“ eine Inputmenge von ca. 0,01 bis $0,04 \mathrm{~kg}$ Nano-Ag pro Jahr für Putztücher zu berechnen. Alle Annahmen und Berechnungsschritte, die für diese Materialflussanalysen notwendig waren, werden im Endbericht des Projektes „NanoMia“ (2015) genauer erläutert. Beim letzten „Szenario S6“ stellte sich im Zuge der Recherchen heraus, dass Quantenpunkte laut Herstellerangaben und wissenschaftlichen Publikationen bereits in hochauflösenden Bildschirmen eingesetzt werden, der derzeitige Stand des Wissens bzw. die verfügbare Datenlage aber keine Abschätzung von eingesetzten Mengen zulässt. Dieses Nanoprodukt ist daher ein Beispiel dafür, dass ein systematischer Informationsmangel hinsichtlich der Produktinhaltsstoffe dazu führt, dass potenzielle Umweltauswirkungen bzw. deren Umwelteintrag derzeit nicht eingeschätzt werden können.

Somit konnten nur für „Szenario S 1 bis S 5“ die österreichweiten Verbrauchsmengen abgeschätzt werden. 
Diese wurden als Inputmengen für die gegenständlichen Materialflussanalysen herangezogen, um nachfolgende Materialflüsse sowohl für die Nutzungsals auch Entsorgungsphase berechnen zu können. Diese Produktlebenszyklusphasen stellten die Systemgrenze der Materialflussanalyse dar (Bezugsjahr 2014); die Produktionsphase wurde aufgrund der nicht verfügbaren Grundlagendaten nicht berücksichtigt. SystemOutputmengen, wie der Export von Nanoprodukten, konnten aufgrund der Datenlage nicht abgeschätzt werden. Des Weiteren war es aufgrund fehlender Informationen nicht möglich, die genaue Nutzungsdauer der jeweiligen Nanoprodukte zu berücksichtigen. Zur Vereinfachung wurde somit angenommen, dass diese Produkte auch im selben Jahr (d. h. in 2014) entsorgt wurden. Senken für die Nutzungsphase - d. h. jene Nanomaterialmengen, die während der Nutzungsdauer in der Produktmatrix verbleiben - konnten aus diesem Grund ebenfalls nicht abgeschätzt werden. Um marktdynamische Prozesse, zeitliche Veränderungen sowie Produktnutzungsdauern entsprechend zu berücksichtigen, könnten für zukünftige Studien sogenannte dynamische Materialflussanalysen angewendet werden (insofern eine fundierte Datengrundlage dies ermöglicht).

\subsection{Nanospezifische}

Transferkoeffizienten

Nach der Abschätzung der Einsatzbzw. System-Inputmengen wurden sogenannte Transferkoeffizienten aus wissenschaftlichen Publikationen herangezogen, um nähere Aussagen hinsichtlich Verteilung und Verbleib von Nanomaterialien in der Umwelt tätigen zu können. Anhand dieser Transferkoeffizienten können die verbleibenden Nanomaterialmengen nach einem bestimmten Prozess (z. B. dem Gebrauch oder Recycling von Nanoprodukten) berechnet werden. Solche Koeffizienten werden häufig durch experimentelle Untersuchungen über die potenziellen Freisetzungsraten von Nanomaterialien - z. B. hervorgerufen durch mechanische oder chemische Einwirkungen bestimmt. Hinsichtlich „Szenario 1“ ist z. B. aus der Studie von Danovaro et al. (2008) bekannt, dass der Großteil bzw. $75 \%$ der Sonnencreme erst durch den Einsatz von Seifen beim Duschen abgewaschen werden. Demnach werden nur ca. $25 \%$ während des Badens (ohne Sei- fen) abgewaschen. Des Weiteren zeigte eine experimentelle Studie von Kaegi et al. (2013), dass Nanomaterialien in der Kanalisation über sehr weite Strecken transportiert werden können. Untersuchungen in einer Versuchskläranlage legten dar, dass ca. $90 \%$ der dotierten Menge an Nano- $\mathrm{TiO}_{2}$ durch den Klärschlamm rückgehalten werden und nur ein geringer Anteil von $10 \%$ diffus in den Vorfluter emittiert wird (Johnson et al. 2011). Gepaart mit der Kenntnis, dass in Österreich ca. 50 \% der kommunalen Klärschlämme thermisch behandelt werden (Anm.: nach dem Verbot der direkten Klärschlammdeponierung in 2004) (Egle et al. 2014), konnte in einem weiteren Schritt der anteilsmäßige Verbleib von Nano- $\mathrm{TiO}_{2}$-haltigen Sekundärabfallströmen in der Umwelt bzw. Abfallwirtschaft abgeschätzt werden (d. h. die Mengenanteile, die zur direkten, landwirtschaftlichen Verwertung oder zur thermischen Behandlung gehen). Zusätzlich ist durch einen in der Schweiz durchgeführten, großmaßstäblichen Versuch über das Verhalten von Nanomaterialien während der Abfallverbrennung bekannt, dass ca. 99,99\% dieser Nanomaterialien in den festen Verbrennungsrückständen wie Schlacken und Flugaschen verbleiben. Im Zuge der gegenständlichen Materialflussanalysen konnte somit die deponierte Masse an $\mathrm{Nano}^{-\mathrm{TiO}_{2}}$ in festen Verbrennungsrückstanden bzw. Reststoffen berechnet werden.

Anhand solcher Nanomaterial-spezifischer Transferkoeffizienten wurde auch der Verbleib von Nano-Ag, $-\mathrm{SiO}_{2}$ und CNTs nach bestimmten, abfallwirtschaftlichen Behandlungsprozessen abgeschätzt, wobei je nach Szenario auf unterschiedliche Studien zurückgegriffen wurde (z. B.: Kaegi et al. 2010; Mitrano et al. 2014; Mueller et al. 2013). Wenn für bestimmte Prozesse keine nanospezifischen Koeffizienten und Freisetzungsraten verfügbar waren, wurde wie bei vergleichbaren Materialflussanalysen - z. B. von Caballero-Guzman and Nowack (2016) oder Gottschalk et al. (2010) - vereinfacht angenommen, dass Nanoabfälle dieselbe Mengenverteilung wie ihre zugehörigen Abfallströme bzw. -fraktionen aufweisen (probabilistischer Ansatz). Aus dem Bundesabfallwirtschaftsplan (BMLFUW 2011) ist beispielsweise bekannt, dass ca. $91 \%$ der getrennt gesammelten Baurestmassen als mineralischer Bauschutt und ca. $9 \%$ als Baustellenabfälle gesammelt werden. Dem probabilisti- schen Ansatz entsprechend verteilt sich beispielsweise in den Baurestmassen enthaltenes Nano-Ag („Szenario 2“) mit jener Wahrscheinlichkeit auf die Stoffströme mineralischer Bauschutt und Baustellenabfällen, die der Massenverteilung der Stoffströme entspricht. In weiterer Folge kann so z. B. die deponierte Menge an Nano-Ag-haltigen Reststoffen aus dem Bauschutt-Recycling vereinfacht abgeschätzt werden. Im Endbericht des Projektes "NanoMia“ (2015) wurden diese nicht nanospezifischen Koeffizienten, welche ausschließlich auf allgemeinen Abfallstatistiken basieren (probabilistischer Ansatz), näher erläutert. Diese Untersuchungen zeigen, dass mithilfe einer Materialflussanalyse und unter Verwendung zahlreicher, vereinfachter Annahmen eine erste quantitative Abschätzung des potenziellen (Umwelt-)Verbleibs von Nanomaterialien in Österreich bei bestimmten Produktgruppen möglich ist. Die großen Streubreiten und Unsicherheiten hinsichtlich Einsatzmengen und potenzieller Umweltverteilung, welche die Materialflussanalysen zeigen, spiegeln wider, dass derzeit nur auf eine sehr beschränkte Anzahl an relevanten Studien sowie experimentellen Untersuchungen über das Freisetzungsverhalten von Nanomaterialien zurückgegriffen werden kann.

\section{Exemplarische Ergebnisse - Verteilung von Nanomaterialien im österreichischen Abfallwirtschaftssystem}

In diesem Kapitel werden exemplarisch anhand zweier ausgewählter Konsumprodukte (aus den sechs untersuchen Produkten) die Materialflussanalysen dargestellt und die Erkenntnisse daraus kurz diskutiert.

In Abb. 2 ist das „Maximum-Szenario S1: $\mathrm{Nano}^{-\mathrm{TiO}_{2}}$ in Sonnencremen“ als Materialflussdiagramm für das Betrachtungsjahr 2014 dargestellt. Jährlich werden schätzungsweise ca. $25 \%$ des Jahresverbrauchs an Nano- $\mathrm{TiO}_{2}$ in Sonnenschutzcremen diffus in Bade- und Fließgewässer ausgetragen und damit nicht von kommunalen Abwasser- bzw. Abfallsammelsystemen erfasst. Derartige Emissionen, die nicht als Abwasser oder Abfall gesammelt werden können, werden in der gegenständlichen $\mathrm{Ma}$ terialflussanalyse als „diffuser Eintrag“ in die Umweltkompartimente Wasser, Boden und Luft gekennzeichnet. Der größte Anteil (ca. 26,6 t) an in Son- 


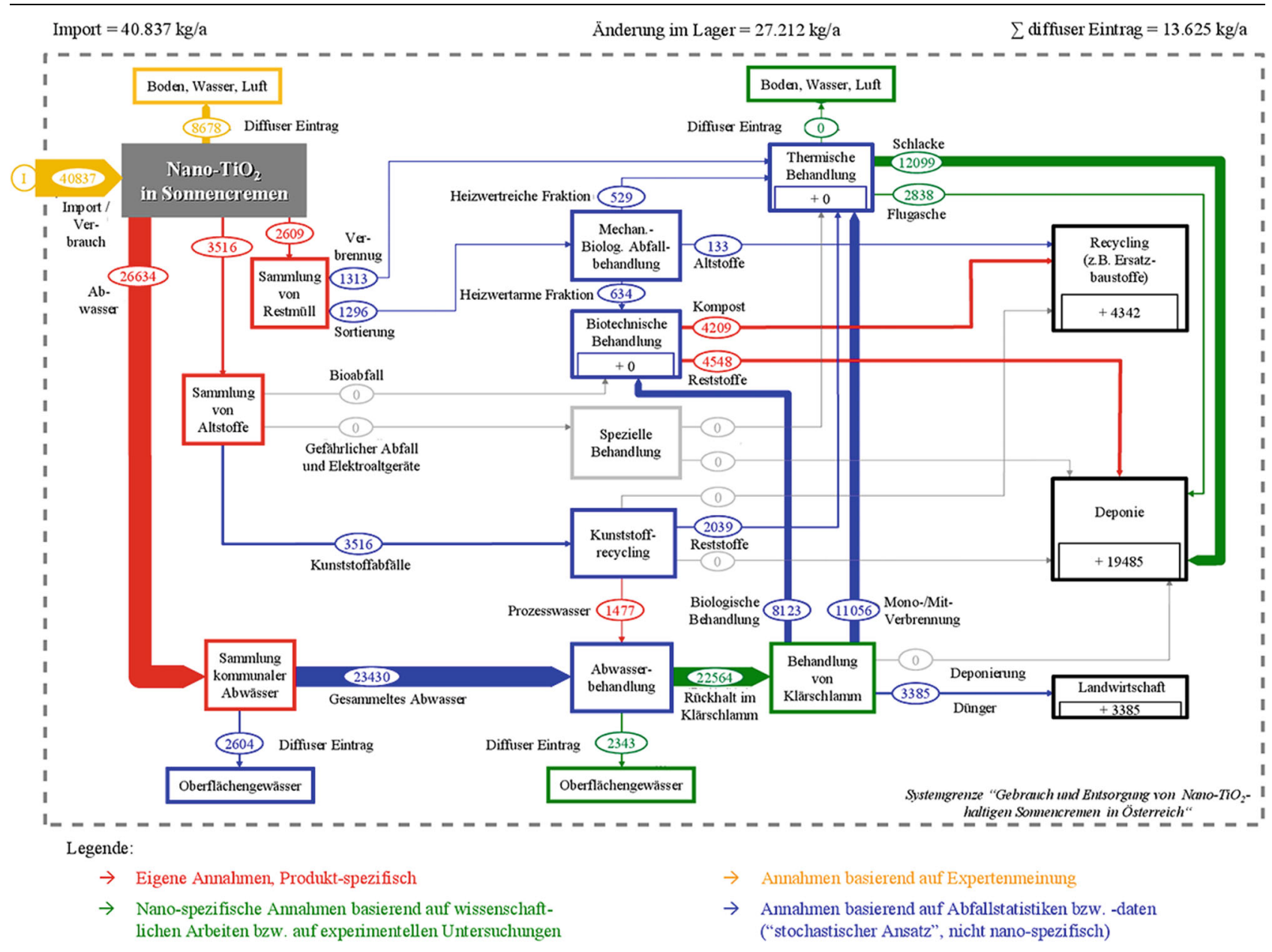

Abb. 2 Materialfluss von Nanotitandioxid in Sonnencremen für das Jahr 2014 („Maximum-Szenario“): Abschätzung von in Österreich jährlich verbrauchtem Nano- $\mathrm{TiO}_{2}$ in Sonnencremen sowie der durch den Gebrauch entstehenden Nanoabfallmengen

nencremen enthaltenem $\mathrm{Nano}_{-} \mathrm{TiO}_{2}$ wird über die kommunale Abwassersammlung erfasst und gelangt in die Abwassereinigung. Mittels der aus der Literatur entnommenen nanospezifischen Transferkoeffizienten konnte abgeschätzt werden, dass ca. $22,5 \mathrm{t}$ Nano- $\mathrm{TiO}_{2}$ während der biologischen Reinigung im Klärschlamm rückgehalten werden. Ein geringer Anteil von ca. 2,3 t wird nach der Abwasserbehandlung diffus in die Vorfluter emittiert. Gemeinsam mit dem Klärschlamm gelangen ca. 3,4 t Nano- $\mathrm{TiO}_{2}$ direkt in die Landwirtschaft (Klärschlammaufbringung), ca. 11,0 t in die thermische Behandlung und ca. 8,3 t in die biotechnische Behandlung (z. B. in Kompostieroder Biogasanlagen). Nach den thermischen Behandlungsschritten verbleibt der Großteil dieser Nanoabfälle in den festen Rückständen und wird somit am Ende deponiert. Ein geringer Anteil an Nano- $\mathrm{TiO}_{2}$ kann über den Altstoffstrom in Form von Recyclingprodukten wie- der in den „anthropogenen Kreislauf“ bzw. über Komposte aus der biotechnischen Behandlung von Klärschlämmen in die Umwelt gelangen.

In Abb. 3 wird ein Nanoprodukt dargestellt, bei welchem Nano-Ag in der Produktmatrix bzw. in Wandfarben in fest gebundener Form (nach Trocknung bzw. Aushärtung) vorliegt. Im Gegensatz zu „Szenario 1“ (bzw. Abb. 2) haben Klärschlämme in diesem Fall keine Bedeutung, da davon ausgegangen wurde, dass während der Nutzungsdauer von Innendispersionen nur ein sehr geringer bis zu vernachlässigender Mengenanteil von Nano-Ag durch Reinigungsarbeiten freigesetzt bzw. ausgewaschen wird. Dieses Szenario zeigt somit auch, dass die Bedeutung von Arbeitssicherheitsaspekten während Recyclingprozesse zukünftig zunehmen wird, da z.B. durch mechanische Beanspruchung während einzelner Aufbereitungsschritte eine unbeabsichtigte Freisetzung von Nanomaterialien stattfinden und somit zu einer erhöhten Exposition führen könnte.

Es kann gemäß Abb. 3 davon ausgegangen werden, dass der Großteil des in Innendispersionen enthaltenen NanoAg (ca. 2,8 kg pro Jahr) als Baurestmassen gesammelt und weiterbehandelt wird. Basierend auf eigenen Einschätzungen wurde angenommen, dass ca. $10 \%$ des Gesamtjahresverbrauchs an Nano-Ag in den zu entsorgenden Farbbehältern verbleiben. Über den Restmüll oder die Altstoffsammlung bzw. teilweise auch Problemstoffsammlung wird somit eine (vergleichsweise sehr geringe) Menge von ca. 0,3 kg Nano-Ag erfasst und weiterbehandelt. Gelangen Ag-haltige Nanoabfälle in eine Müllverbrennungsanlage, wird von Müller et al. (2013) davon ausgegangen, dass ca. 2,5 \% der jeweiligen Inputmengen während der Rauchgasreinigung durch saure Wäscher in z. B. Silberchlorid oder gelöste Silberionen umgewandelt werden. Im Zuge der Behandlung der ein- 


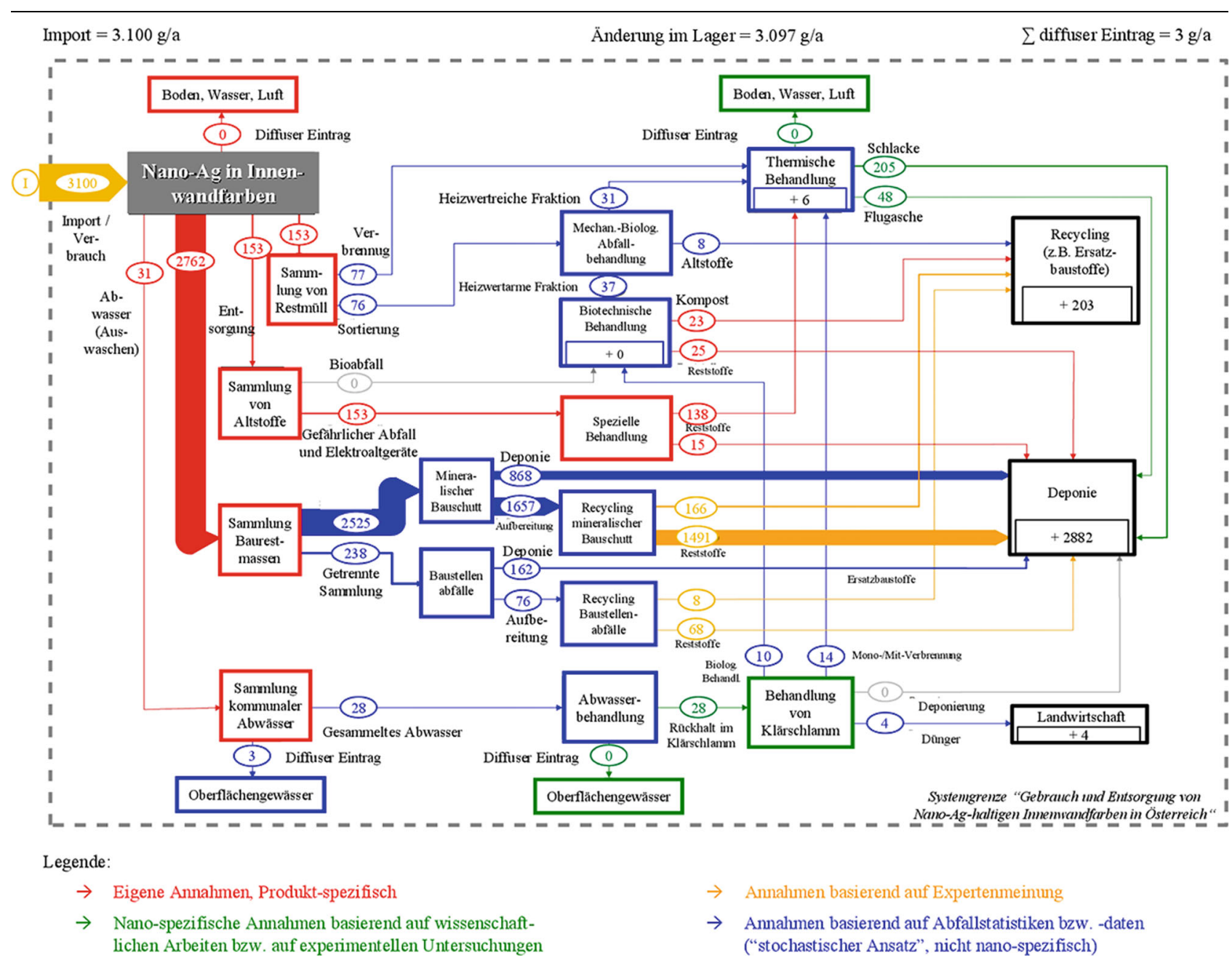

Abb. 3 Materialfluss von Nanosilber in Innenwandfarben für das Jahr 2014 („,Maximum-Szenario“): Abschätzung von in Österreich jährlich verbrauchtem Nano-Ag in Innenwandfarben sowie der durch den Gebrauch entstehenden Nanoabfallmengen

zelnen Abfallströme gelangt der Großteil des Nano-Ag (ca. 2,9 kg) aus Innenwandfarben schlussendlich über feste Reststoffe/Behandlungsrückstände auf Deponien. Etwa die Hälfte des deponierten Nano-Ag ist dabei in den Reststoffen aus dem Recycling von mineralischem Bauschutt enthalten.

In der folgenden Abb. 4 werden alle Ergebnisse der Abschätzung des Umweltverbleibs der sechs im Rahmen dieser Studie ausgewählten Nanomaterialien bzw. -produkten in Österreich zusammenfassend dargestellt. Die durchgeführten Materialflussanalysen verdeutlichen, dass sich Nanomaterialien in den meisten Szenarien vorwiegend in Deponien vor allem über Reststoffe, welche Nanopartikel enthalten und nach den jeweiligen Behandlungsschritten deponiert werden, aber auch in Recyclingprodukten anreichern. Die Abfallwirtschaft (wie auch Abwasser- wirtschaft) spielt somit eine entscheidende Rolle, um unkontrollierte, diffuse wasser- oder luftseitige Emissionen (z. B. nanomaterialhaltige Kläranlagenabflüsse oder Rauchgase während der Abfallverbrennung) zu verhindern. Gemäß „Szenario 3“ kann bei der Entsorgung von CNTs in Tennisschlägern davon ausgegangen werden, dass beinahe $100 \%$ der entsorgten Mengen während der thermischen Behandlung eliminiert bzw. zu $\mathrm{CO}_{2}$ oxidiert werden (Müller et al. 2013). Hierzu ist jedoch anzumerken, dass eine vollständige Elimination von CNTs nur dann gewährleistet werden kann, wenn die stöchiometrischen Bedingungen während der Abfallverbrennung dies auch zulassen. So kann laut Ounoughene et al. (2015) eine vollständige Oxidation von CNTs nur dann sichergestellt werden, wenn sehr hohe Temperaturen $\left(>850^{\circ} \mathrm{C}\right)$ über eine bestimmte Verweildauer eingehalten werden.

\section{Schlussfolgerungen und Ausblick}

Ohne verlässliche Informationen über Produktionsmengen von Nanomaterialien sowie deren genauen Einsatz in Produkten (Marktanteile, stoffliche Zusammensetzung, Inhaltsmenge etc.) ist eine belastbare Mengenfestlegung der Verteilung von Nanomaterialien in der Anthroposphäre und Umwelt kaum möglich. Um trotzdem eine erste grobe Einschätzung einer möglichen Verteilung von Nanomaterialien in der österreichischen Abfallwirtschaft zu erhalten, wurden für sechs ausgewählte Konsumprodukte vereinfachte Materialflussanalysen (für Gebrauchsphase und Entsorgungsphase) durchgeführt. Dabei konnte teilweise auf nanomaterialspezifische Transferkoeffizienten aus 


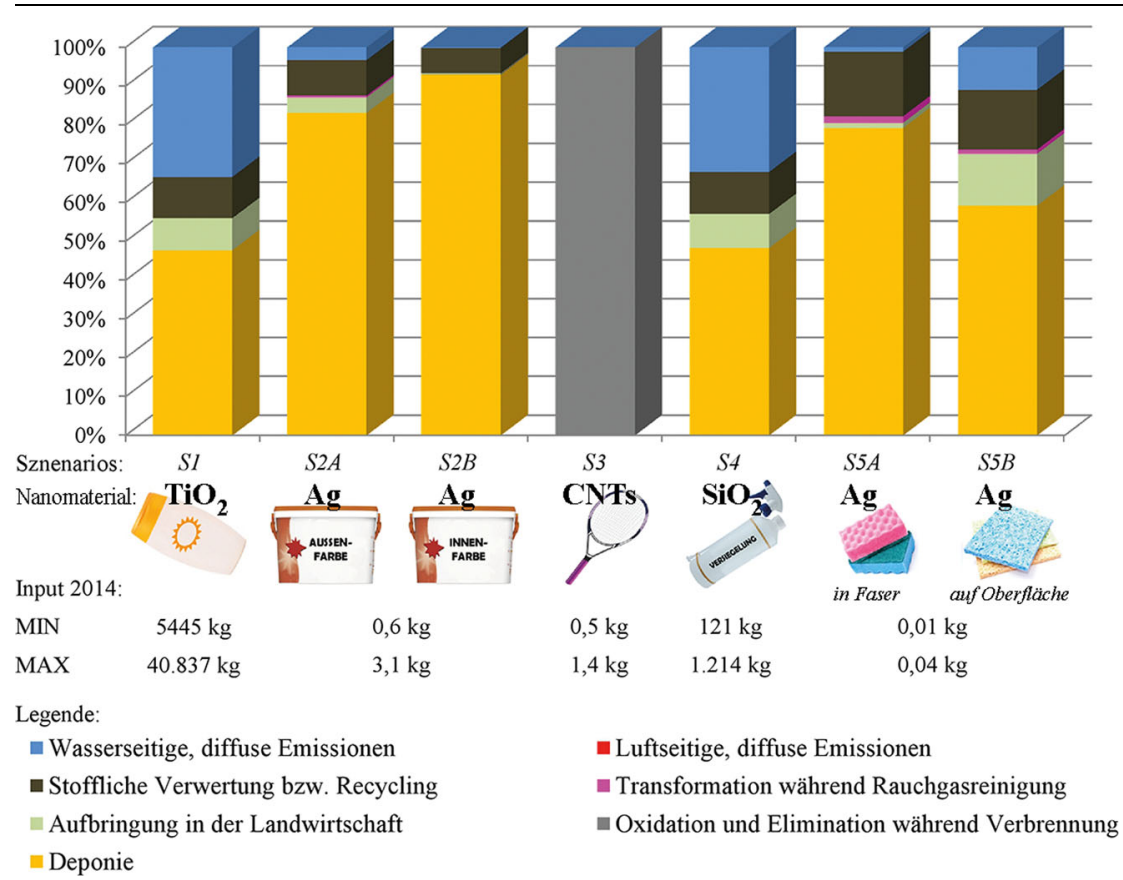

Abb. 4 Potenzieller Umweltverbleib von Nanomaterialien aus ausgewählten österreichischen Konsumprodukten-Zusammenfassung der Ergebnisse aus den durchgeführten Materialflussanalysen („,Szenarien S1 bis S 5“)

der Literatur zurückgegriffen werden. Derzeit sind vereinzelte Studien und/ oder experimentelle Untersuchungen verfügbar, die zum Ziel hatten, Freisetzungsraten bzw. Transferkoeffizienten von Nano-Ag, $-\mathrm{SiO}_{2},-\mathrm{TiO}_{2}$ oder CNTs während der Abwasserreinigung oder Abfallverbrennung zu bestimmen. Über das genaue Umweltverhalten von Nanomaterialien während abfallwirtschaftlicher Behandlungsprozesse, wie mechanisch-biologische Behandlung oder chemisch/physikalische Recyclingverfahren, ist bis dato sehr wenig bekannt. So musste im Zuge der Materialflussanalysen aufgrund dieser fehlenden Daten ein sogenannter probabilistischer Ansatz angewendet werden, der auf öffentlich zugänglichen Abfallstatistiken basiert. Hierbei wurde davon ausgegangen, dass der Materialfluss eines nanomaterialhaltigen Abfallstroms gleich aufgeschlüsselt ist wie jener ohne Nanomaterial. Dieser probabilistische Ansatz stellte sich als unabdingbar heraus, um überhaupt erste Massenflüsse für Nanoabfälle bilanzieren zu können. Diese für Österreich ersten, vereinfachten Materialflussanalysen haben gezeigt, dass die größten Mengen an metallischen/metalloxidischen Nanomaterialien über Sekundärabfälle, wie Klärschlämme oder Reststoffe aus der Abfallverbrennung, am Ende in Depo- nien gelangen und sich dort anreichern können. Diese Ergebnisse stimmen mit vergleichbaren, internationalen Materialflussanalysen anderer Autoren überein, welche festgestellt haben, dass Deponien offensichtlich für Nanomaterialien eine wichtige Senke darstellen. Berücksichtigen muss man allerdings, dass es noch kaum Untersuchungen zum genauen Verbleib bzw. über das wasser- oder luftseitige Freisetzungsverhalten von Nanomaterialien in Denoch nicht verlässlich beantwortet werden, ob z. B. Deponiebasisdichtungen oder vorherrschende Sorptionskapazitäten den Austrag von Nanomaterialien (langfristig) unterbinden können. Aus den österreichischen Szenarien lässt sich ebenfalls ableiten, dass ein geringer Mengenanteil an metallischen Nanomaterialien vermutlich derzeit über die stoffliche Verwertung in Recyclingmaterialien transferiert wird bzw. durch Komposte in die Umwelt gelangen kann. Hinsichtlich kohlenstoffbasierter Nanomaterialien lässt sich vom derzeitigen Wissensstand ableiten, dass diese in der Entsorgungsphase - sofern sie einer entsprechenden thermischen Behandlung bei ausreichenden Temperaturen $\left(>850{ }^{\circ} \mathrm{C}\right)$ unterzogen werden - vollständig oxidieren und somit eliminiert werden. Ihr Verhalten während ponien gibt. Das heißt, es kann derzeit der Abfallverbrennung ist allerdings sehr stark von den stöchiometrischen Bedingungen, der Temperatur sowie der Verweildauer abhängig.

Generell ist jedoch die Menge an Nanoabfällen, die derzeit in die österreichische Abfallwirtschaft eingebracht werden, im Vergleich zu den herkömmlichen Abfallströmen äußerst gering. Wie aber bereits eingangs erwähnt, spielt im Zusammenhang mit Nanomaterialien nicht unbedingt die "Masse“, sondern eher deren sehr reaktives und derzeit bei Risiko- und Umweltbetrachtungen noch schwer einschätzbares Verhalten die wesentlich wichtigere Rolle. Die Ergebnisse der durchgeführten Materialflussanalysen verdeutlichen allerdings auch, dass - solange Abfälle und Abwässer sachgerecht gesammelt und behandelt werden - diffuse, unkontrollierte Emissionen von Nanomaterialien gering gehalten werden. Dennoch ist eine potenzielle Freisetzung von synthetisierten Nanomaterialien während abfalltechnischer Behandlungsprozesse aufgrund von mechanischen, chemischen und thermischen Einwirkungen möglich. Dies muss in Zukunft vor allem auch hinsichtlich Arbeitssicherheit und Gesundheitsschutz für Beschäftigte in der Abfallwirtschaft (z. B. in Recyclinganlagen) noch verstärkt beobachtet und berücksichtigt werden. In diesem Zusammenhang wird darauf hingewiesen, dass die Detektion und Charakterisierung von Nanoobjekten (Nanopartikel, Nanofasern etc.) in komplexen Umweltproben, wie flüssigen oder festen Abfällen, sehr aufwendig und kostenintensiv ist, da bis dato zum Umwelt- oder Abfallmonitoring noch kaum routinemäßige und praxisgerechte Messmethoden zur Verfügung stehen. So sind für eine umfassende, objektive Beurteilung des Umweltverhaltens von Nanomaterialien prinzipiell sowohl partikel- wie auch matrixbezogene Parameter zu ermitteln (z. B. Partikelgröße und -verteilung, Form, Struktur und spezifische Oberflächeneigenschaften bzw. pH-Wert, Ionenstärke sowie Gehalt an organischen Substanzen). Zur Bestimmung dieser Parameter werden in der Wissenschaft derzeit unterschiedliche Messmethoden kombiniert (z. B. auf Massenspektrometrie basierende Verfahren oder Streumethoden in Kombination mit bildgebenden Verfahren wie Elektronenmikroskopie), welche auf Abfallproben erst angepasst und in weiterer Folge standardisiert werden müssen (Part et al. 2015). 
Zusammengefasst dargestellt, ermöglichen die durchgeführten und aufgrund der Datenlage stark vereinfachten Materialflussanalysen etwaige „Emissionshotspots“ (am Beispiel der ausgewählten Konsumprodukte) in der österreichischen Abfallwirtschaft $\mathrm{zu}$ identifizieren. Hierbei kann z. B. abgeschätzt werden, ob bestimmte Nanomaterialien potenziell schon in der Gebrauchsphase oder erst in der Abfallbehandlungs- bzw. Entsorgungsphase freigesetzt werden können. Diese Informationen sind wichtig, um in $\mathrm{Zu}$ kunft gezielt spezifische Messungen und Kontrollmechanismen einsetzen zu können. Die gegenständliche Studie hat vor allem gezeigt, dass derzeit sehr wenige experimentelle Untersuchungen über potenzielle Freisetzungsraten bzw. -pfade von Nanomaterialien - insbesondere in der Entsorgungsphase verfügbar sind. Solche Untersuchungen sind aber notwendig, um zuverlässige nanomaterialspezifische Transferkoeffizienten oder Modellparameter (z. B. Transfer von festen nanohaltigen Abfällen in wässrige Phasen oder nanospezifische Löslichkeits-/Aggregationsraten) ableiten zu können und somit potenzielle Risiken, die von synthetisch hergestellten Nanomaterialien ausgehen können, auch objektiv beurteilen zu können.
Open access funding provided by University of Natural Resources and Life Sciences Vienna (BOKU).

Open Access Dieser Artikel wird unter der Creative Commons Namensnennung 4.0 International Lizenz (http:// creativecommons.org/licenses/by/4. 0/deed.de) veröffentlicht, welche die Nutzung, Vervielfältigung, Bearbeitung, Verbreitung und Wiedergabe in jeglichem Medium und Format erlaubt, sofern Sie den/die ursprünglichen Autor(en) und die Quelle ordnungsgemäß nennen, einen Link zur Creative Commons Lizenz beifügen und angeben, ob Änderungen vorgenommen wurden.
Aboulaich, A., Balan, L., Ghanbaja, J., Medjahdi, G., Merlin, C., Schneider, R. (2011): Aqueous Route to Biocompatible ZnSe:Mn/ZnO Core/ Shell Quantum Dots Using 1-Thioglycerol As Stabilizer. Chemistry of Materials 23, 3706-3713. AGES (2016): NanoInformationsPortal. Homepage. Österreichische Agentur für Gesundheit und Ernährungssicherheit GmbH. http:// nanoinformation.at/ (letzter Zugriff am 12.9.2016). Anikeeva, P. O., Halpert, J. E., Bawendi, M. G. Bulović, V. (2009): Quantum Dot Light-Emitting Devices with Electroluminescence Tunable over the Entire Visible Spectrum. Nano Letters 9 , 2532-2536.

BMLFUW (2011): Bundesabfallwirtschaftsplan 2011, Band 1, Bundesministerium für Land- und Forstwirtschaft, Umwelt und Wasserwirtschaft, Wien.

Boldrin, A., Hansen, S. F., Baun, A., Hartmann, N., Astrup, T. (2014): Environmental exposure assessment framework for nanoparticles in solid waste. J Nanopart Res 16, 1-19.

Caballero-Guzman, A, Nowack, B. (2016): A critical review of engineered nanomaterial release data: Are current data useful for materia flow modeling? Environmental Pollution 213, 502-517.

Danovaro, R., Bongiorni, L., Corinaldesi, C. Giovannelli, D., Damiani, E., Astolfi, P., Greci, L., Pusceddu, A. (2008): Sunscreens Cause Coral Bleaching by Promoting Viral Infections. Environmental Health Perspectives 116, 441-447.

Davis, J. M., Long, T. C., Shatkin, J. A., Wang, A., Grahm, J. A., Gwinn, M., Ranalli, B. (2010): Nanomaterial Case Studies: Nanoscale Titanium Dioxide in Water Treatment and in Topical Sunscreen. EPA/600/R-09/057F U.S. Environmental Protection Agency, Research Triangle Park, NC. Egle, L., Rechberger, H., Zessner, M. (2014) Endbericht. Phosphorbilanz Österreich. Grundlage für ein nachhaltiges Phosphormanagement
- gegenwärtige Situation und zukünftige Entwicklung. Bundesministerium für Land- und Forstwirtschaft, Umwelt und Wasserwirtschaft, Sektion VII - Wasserwirtschaft, Wien.

Gazsó, A., Fuchs, D., Greßler, S., Gruber, I., Part F., Huber-Humer, M. (2015): Nanotechnology Environmentally Relevant Aspects of Nanomaterials in Products at the End-of-life Phase. European Journal of Risk Regulation 6 .

Gottschalk, F., Scholz, R.W., Nowack, B. (2010): Probabilistic material flow modeling for assessing the environmental exposure to compounds: Methodology and an application to engineered nano-TiO2 particles. Environmental Modelling \& Software 25, 320-332.

Greßler, S., Gazsó, A. (2014): Nano-Konsumprodukte in Österreich - Aktualisierung und Adaptierung der NanoTrust-Datenbank (NanoTrustDossier Nr. 041 - November 2014). Institut für Technikfolgen-Abschätzung (ITA), Wien.

ISO/TS 80004-1:2015: Nanotechnologies - Vocabulary - Part 1: Core terms. International Organization for Standardization (ISO).

Johnson, A. C., Bowes, M. J., Crossley, A., Jarvie, H. P., Jurkschat, K., Jürgens, M. D., Lawlor, A. J. Park, B., Rowland, P., Spurgeon, D., Svendsen, C., Thompson, I, P, Barnes, R. J., Williams, $R$ J., Xu, N. (2011): An assessment of the fate, behaviour and environmental risk associated with sunscreen TiO2 nanoparticles in UK field scenarios. Science of The Total Environment 409, 2503-2510.

Kaegi, R., Sinnet, B., Zuleeg, S., Hagendorfer, H., Mueller, E., Vonbank, R., Boller, M., Burkhardt, M. (2010): Release of silver nanoparticles from outdoor facades. Environmental Pollution $158,2900-2905$

Kaegi, R., Voegelin, A., Ort, C., Sinnet, B., Thalmann, B., Krismer, J., Hagendorfer, H., Elumelu, M., Mueller, E. (2013): Fate and transforma- tion of silver nanoparticles in urban wastewater systems. Water Research.

Krug, H. F. (2014): Nanosicherheitsforschung sind wir auf dem richtigen Weg? Angew Chem Int Ed Engl 126, 12502-12518.

Lorenz, C., Von Goetz, N., Scheringer, M., Wormuth, M., Hungerbuhler, K. (2011): Potential exposure of German consumers to engineered nanoparticles in cosmetics and personal care products. Nanotoxicology 5, 12-29.

Mitrano, D.M., Rimmele, E., Wichser, A., Erni, R., Height, M., Nowack, B. (2014):. Presence of Nanoparticles in Wash Water from Conventional Silver and Nano-silver Textiles. ACS Nano 8 7208-7219.

Mueller, N. C., Buha, J., Wang, J., Ulrich, A., Nowack, B. (2013): Modeling the flows of engineered nanomaterials during waste handling. Environmental Science: Processes \& Impacts 15 251-259.

NanoMia (2015): NanoMia. Nanomaterialien in Abfällen: Evaluierung von Regelungen und Verfahren zum Nachweis von Nanomaterialien in Abfallströmen. Endbericht des Projektes 844415. Institut für Abfallwirtschaft, Universität für Bodenkultur Wien \& Institut für TechnikfolgenAbschätzung der Österreichischen Akademie der Wissenschaften

Ounoughene, G., Le Bihan, O., Chivas-Joly, C., Motzkus, C., Longuet, C., Debray, B., Joubert, A., Le Coq, L., Lopez-Cuesta, J. M. (2015): Behavior and Fate of Halloysite Nanotubes (HNTs) When Incinerating PA6/HNTs Nanocomposite. Environ Sci Technol 49, 5450-5457.

Part, F., Zecha, G., Causon, T., Sinner, E.-K., Huber-Humer, M. (2015): Current limitations and challenges in nanowaste detection, characterisation and monitoring. Waste Management 43, 407-420. 